\title{
Successful management of acute renal failure with a vaginal pessary: a case report
}

\author{
John P. Micha • Mark A. Rettenmaier • \\ Matthew Clark • Jim C. Hu • Miles Chang • \\ John V. Brown III • Bram H. Goldstein
}

Received: 6 April 2007 / Accepted: 14 June 2007 / Published online: 12 July 2007

(C) Springer-Verlag 2007

\begin{abstract}
Persistent uterine prolapse with secondary acute renal failure is a very uncommon event. We report the case of a 77-year-old woman with a 5-year history of uterine prolapse. She was referred to our gynecologic oncology service for 3rd degree uterine prolapse and was found to have bilateral hydronephrosis and acute renal insufficiency. The patient was fitted for a pessary to manage the uterine prolapse, which resulted in resolution of her hydronephrosis and renal insufficiency. Prompt assessment with nephrology consultation and pessary placement for patients with 3rd degree genital prolapse is imperative to ensure that irreversible renal complications do not manifest themselves.
\end{abstract}

This study was supported by a grant from the Women's Cancer Research Foundation.

J. P. Micha $(\bowtie) \cdot$ M. A. Rettenmaier $\cdot$ J. V. Brown III

B. H. Goldstein

Gynecologic Oncology Associates,

Hoag Memorial Hospital Cancer Center,

351 Hospital Road, Suite 507,

Newport Beach, CA 92663, USA

e-mail: bram@gynoncology.com

\section{Clark}

Department of Obstetrics and Gynecology,

Hoag Memorial Hospital Presbyterian,

Newport Beach, CA, USA

J. C. Hu

Division of Urology, Brigham and Women's Hospital, Boston, USA

\section{Chang}

Department of Radiology, Hoag Memorial Hospital Presbyterian, Newport Beach, CA, USA
Keywords Uterine prolapse · Pessary · Renal failure · Hydronephrosis

\section{Introduction}

Pelvic organ prolapse can be a common and frustrating condition that afflicts many women [4]. The pessary has been successfully employed for the management of uterine prolapse and several additional gynecologic maladies, including cystocele and rectocele $[1,7,10]$.

First- and second-degree uterine prolapses are usually managed with a ring or donut pessary $[1,6,10]$. A cube or gehrung pessary is indicated for patients with 3rd degree or severe uterine prolapse, particularly when a cystocele or rectocele is involved [6].

Third-degree uterine prolapse can be associated with acute and chronic renal failure, particularly if left untreated $[2,5,8,9]$. Primarily, the associated renal failure is attributed to descensus-induced bilateral ureteral kinking and obstruction. However, these urological deficits are infrequently reported and may initially be undetected (Sudhakar et al. 2001). We present a patient with a history of persistent uterine prolapse who developed secondary acute renal failure.

\section{Case report}

A 77-year-old (gravida 3, para 3) woman was referred to our practice because of 3rd degree genital prolapse in January 2006. The patient's serum creatinine was $2.4 \mathrm{mg} / \mathrm{dL}$ and BUN $60 \mathrm{mg} / \mathrm{dl}$, indicative of renal insufficiency. 
Her medical history included a simple left ovarian cyst removed in 2003 and CNS surgery for an aneurysm in 1977. The patient's most recent CA-125, PAP smear, and endometrial biopsy were all within normal limits in August 2005.

The patient was admitted to the hospital for medical evaluation of her renal insufficiency. Nephrology and gynecologic urology consultations were obtained. Bilateral renal ultrasound revealed moderate bilateral hydronephrosis and hydro-ureters. No mass or calculi were noted (Fig. 1a and b).

A review of her records indicated that she had a 5-year history of uterine descensus. A few days prior to admission, this progressed to 3 rd degree level prolapse with the cervix and uterus protruding $5-6 \mathrm{~cm}$ beyond the introitus. There was mild cervical/vaginal excoriation and abrasion. The gynecologic urologist placed a no. 4 ring support vaginal pessary to manage the pelvic organ prolapse.

The patient had a comprehensive metabolic panel at the end of January 2006, which demonstrated improvement in both her BUN $(36 \mathrm{mg} / \mathrm{dl})$ and serum creatinine $(1.8 \mathrm{mg} / \mathrm{dl})$. Her acute renal status continued to improve markedly. A
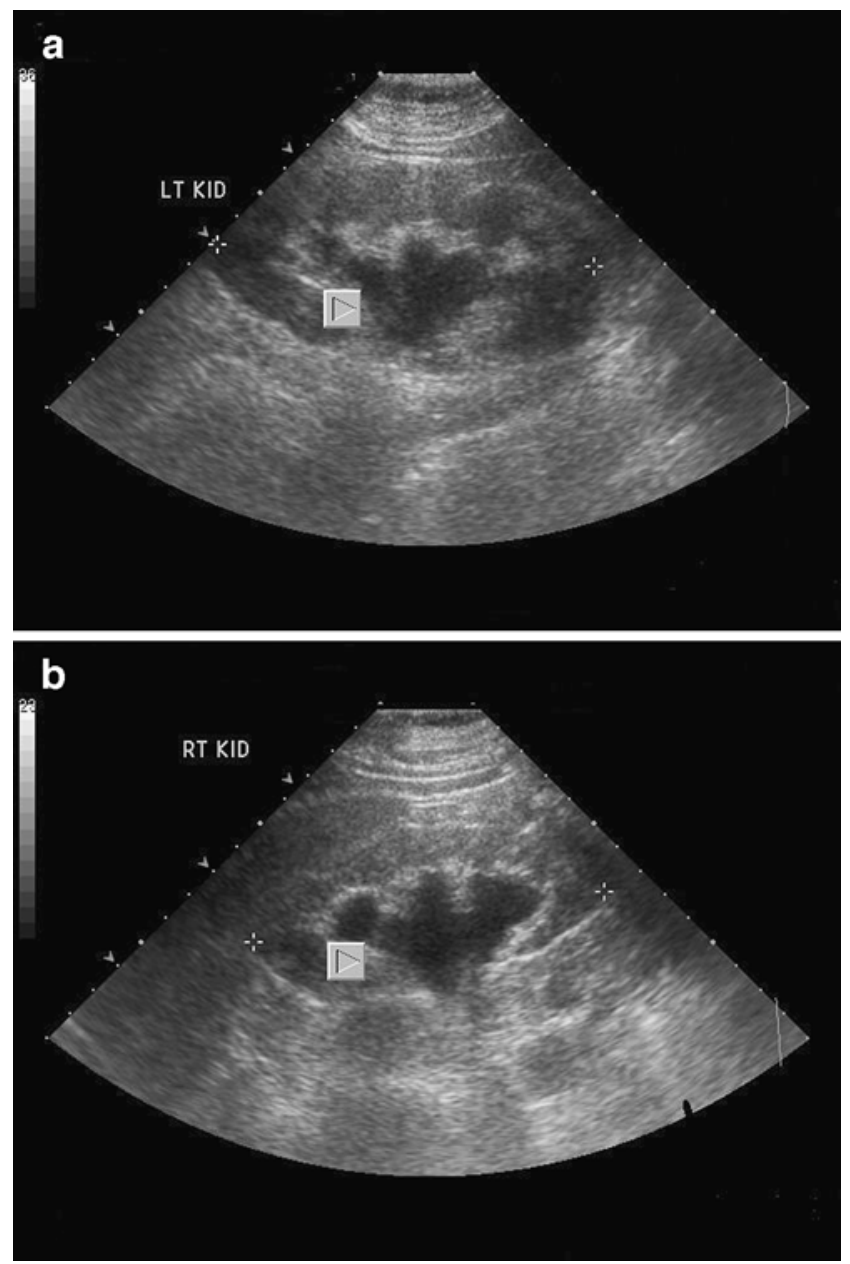

Fig. 1 a and b. Longitudinal renal ultrasound images demonstrating bilateral hydrouretero-nephrosis at presentation
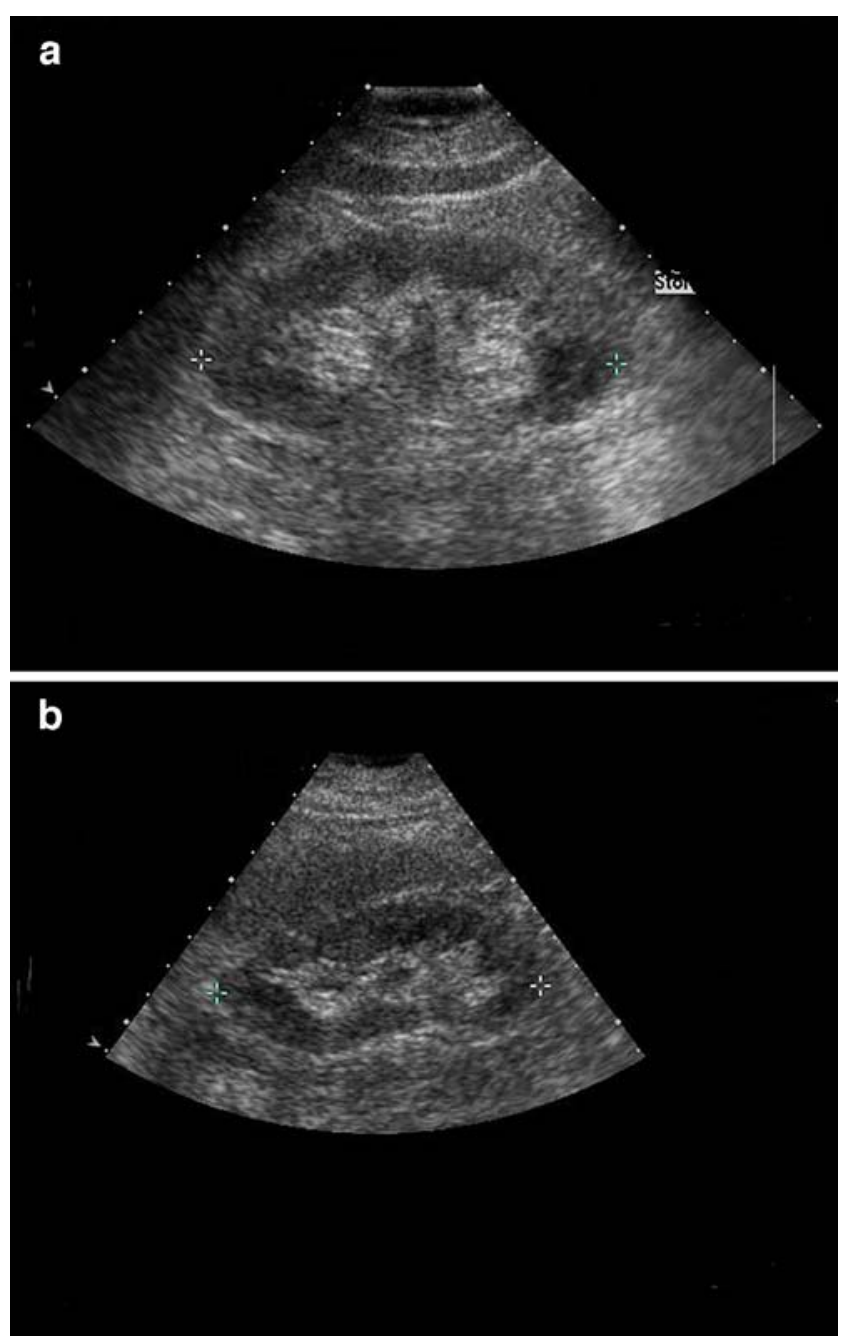

Fig. 2 a and $\mathbf{b}$. Renal ultrasound images demonstrating resolution of hydrouretero-nephrosis post pessary placement

subsequent renal ultrasound demonstrated resolution of her hydronephrosis (Fig. 2a and b). Currently, she is doing very well. A further decision regarding surgical repair of the genital prolapse will be made following consultation with the patient and family.

\section{Discussion}

Uterine organ prolapse with secondary renal failure is very uncommon, with few reported studies detailing the urological sequelae $[2,5]$. Since even incidental uterine prolapse can cause hydronephrosis and ureteral obstruction [2], timely diagnosis and appropriate treatment are imperative to avert potentially high patient morbidity [8].

A review of the literature has identified a select group of current cases of uterine prolapse and secondary renal failure [2, 5, 9, 11]. Chuang et al. [2] discussed two women with uterine prolapse who developed hydronephrosis. Following 
diagnosis and surgery to correct the obstruction, patient renal insufficiency was resolved. Peces et al. [5] discussed the outcome of a 72-year-old woman with acute and chronic renal failure secondary to uterine prolapse. A vaginal pessary was used to reduce the prolapse, and a hysterectomy was performed to further improve the patient's symptoms. In the present study, a ring pessary was used to remedy the patient's prolapse, which ultimately corrected the ureteral obstruction and concomitant renal insufficiency.

Gynecologists rarely encounter patients with acute renal failure secondary to uterine prolapse. Nephrology consultation should be obtained since a delayed or improper diagnosis can result in significant morbidity [3, 8]. If present, pessary reduction may relieve obstructive uropathy, but surgical repair of the prolapse may be indicated $[5,9]$. Furthermore, placement of bilateral nephrostomy tubes may be indicated to salvage an obstructed kidney.

Ideally, renal insufficiency should be reversed prior to prolapse repair in order to mitigate the risk of chronic renal failure. Moreover, transient intra-operative hypotension or other surgical complications will be better tolerated if renal function is optimized preoperatively. Pessary and/or surgical management may comprise optimal patient management $[2$, $3,9]$, although routine treatment has not been determined.

\section{References}

1. Cespedes RD, Cross CA, McGuire EJ (1998) Pelvic prolapse: diagnosing and treating uterine and vaginal vault prolapse. Medscape Womens Health 3:3

2. Chuang FR, Lee CH, Chen CS, Weng HH, Wang IK (2003) Bilateral moderate hyrdroureteronephrosis due to uterine prolapse: two case reports and review of the literature. Ren Fail 25:879-884

3. Churchill DN, Afridi S, Dow D, McManamon P (1980) Uterine prolapse and renal dysfunction. J Urol 124:899-900

4. Palumbo MV (2000) Pessary placement and management. Ostomy Wound Manage 46:40-45

5. Peces R, Canora J, Venegas JL (2005) Chronic renal failure secondary to uterine prolapse. Nefrologia 25:191-194

6. Pott-Grinstein E, Newcomer JR (2001) Gynecologists' patterns of prescribing pessaries. J Reprod Med 46:205-208

7. Ross JW (1997) Techniques of laparoscopic repair of total vault eversion after hysterectomy. J Am Assoc Gynecol Laparosc 4:173-183

8. Sasso K, Hanson L, Smith D (2003) Challenges of pessary management. J Wound Ostomy Continence Nurs 30:152-158

9. Sudhakar As, Reddi VG, Schein M, Gerst PH (2001) Bilateral hyrdroureter and hydronephrosis causing renal failure due to a procidentia: a case report. Int Surg 86:173-175

10. Viera AJ, Larkins-Pettigrew M (2000) Practical use of the pessary. Am Fam Physician 61:2719-2726

11. Yannik FF, Akpolat T, Kocak I (1998) Acute renal failure-an unusual consequence of uterine prolapse. Nephrol Dial Transplant $13: 2648-2650$ 